\title{
PATH INTEGRAL APPROACH TO TWO-DIMENSIONAL QCD IN THE LIGHT-FRONT
}

\author{
P. GAETE \\ Instituto de Física, Universidade Federal do Rio de Janeiro \\ C.P 68528, BR-21945, R.J., Brazil \\ J. GAMBOA ${ }^{\dagger}$ \\ Fachbereich 7 Physik, Universität Siegen, Siegen \\ D-57012, Germany \\ I. SCHMIDT* \\ Departamento de Física, Universidad Técnica Federico Santa Maria \\ Casilla 110-V, Valparaiso, Chile
}

\begin{abstract}
Two-dimensional quantum chromodynamics in the light-front frame is studied following hamiltonian methods. The theory is quantized using the path integral formalism and an effective theory similar to the Nambu-Jona Lasinio model is obtained. Confinement in two dimensions is derived by analyzing directly the constraints in the path integral.
\end{abstract}

Si-93-10

USM-TH-63

IF/UFRJ/93/11

October 1993

$\dagger$ Supported by Alexander von Humboldt Foundation.

* Work supported in part by FONDECYT (Chile), contract 1931120. 


\section{Introduction}

Quantum field theory quantized in the light-front has been extensively studied in the past few years as an alternative way for understanding non-perturbative phenomena [1]. Although this approach is quite old [2], only recently new techniques of calculation have been developed $[3,4]$ that could allow, in principle, the study of phenomena such as confinement or hadronization, which are very difficult to understand through the conventional approach.

Several years ago, t'Hooft studied the solubility of QCD in two dimensions $\left(Q C D_{2}\right)$ in the light-front frame, introducing the $\frac{1}{N}$ expansion [5]. In his work he was able to solve the theory in the large $N$ limit, and then show how the bound state spectrum can be obtained by solving a Bethe-Salpeter equation in this limit. However, in spite of the relevance that the t'Hooft results could have in our understanding of QCD in four dimensions, not much further progress was reached at that time in order to understand the perturbative and non-perturbative structure of QCD in the light-front frame.

The revival of light-front quantization has been mainly pioneered by the authors of reference [3], and later also by those of reference [4]. In these references two different non-perturbative methods for calculating light-front wave functions have been proposed. These wave functions are the amplitudes for a Fock state expansion of the hadronic bound states, in a Hamiltonian light-front approach to QCD. Both methods although promising still present some technical difficulties despite intense recent research [1].

The canonical structure of QCD in the light-front frame and his subsequent quantization via the path integral method is to our knowledge still an open problem. ${ }^{1}$ In the past, this approach has been very useful in the understanding of many aspects of gauge theories and we will show that it is very useful in the present context as well.

The purpose of this research is the study of the canonical structure of light-front

${ }^{1}$ In reference [6] the Hamiltonian formulation of the Schwinger model is studied at the classical level. In this sense, this reference is a particular case of our results. On the other hand, in reference [7] the reader can find recent canonical studies of two dimensional light-front gauge theories. 
$Q C D_{2}$ and its quantization following the path integral method. Our main results are: (a) we find the complete canonical structure (constraints and algebra), (b) the effective field theory obtained after the integration of the gauge field is derived from first principles, and (c) we justify some results obtained by other authors using different methods.

The article is organized as follows: in section 2 the hamiltonian formulation is carried out. In section 3 we discuss the problems asociated with gauge fixing and we quantize the theory following the path integral method. Finally in section 4 we present some conclusions. 


\section{2. $Q C D_{2}$ in Light-Front Coodinates: Hamiltonian}

\section{Analysis}

In this section we study the canonical structure of $Q C D_{2}$ in light-front coordinates following the Dirac constrained theory [8].

Let us start by considering the lagrangian density

$$
\mathcal{L}=-\frac{1}{4} F_{\mu \nu}^{a} F^{a \mu \nu}+\bar{\psi}^{r}(i \not D-m) \psi^{r}
$$

$\left(\mu=0,1 ; a=1,2, \ldots, N^{2} ; r=1,2, \ldots, N\right)$

where the sum convention is assumed. Here $\psi^{r}$ are the $r$ quarks fields and the strenght tensor $F_{\mu \nu}^{a}$ and the covariant derivative are defined as

$$
\begin{aligned}
& F_{\mu \nu}^{a}=\partial_{\mu} A_{\nu}^{a}-\partial_{\nu} A_{\mu}^{a}-g f^{a b c} A_{\mu}^{b} A_{\nu}^{c} \\
& D_{\mu}=\partial_{\mu}+i g A_{\mu}^{a} T^{a}
\end{aligned}
$$

where $g$ is the coupling constant and $T^{a}$ and $f^{a b c}$ are the generators and structure constants associated with the gauge group $S U(N)$.

In the light-front frame approach one defines the coordinates

$$
x^{ \pm}=\frac{1}{\sqrt{2}}\left(x^{0} \pm x^{1}\right)
$$

and then writes all the quantities involved in the lagrangian (2.1) in terms of $x^{ \pm}$instead of $x^{0}$ and $x^{1}$. After doing this, the lagrangian density (2.1) becomes

$$
\mathcal{L}=\frac{1}{2}\left(F_{+-}^{a}\right)^{2}+\bar{\psi}^{r}\left(i \gamma_{-} D_{+}+i \gamma_{+} D_{-}-m\right) \psi^{r}
$$

where $\gamma_{ \pm}$and $D_{ \pm}$are defined in complete analogy with (2.4), i.e

$$
\begin{aligned}
& \gamma_{ \pm}=\frac{1}{\sqrt{2}}\left(\gamma_{0} \pm \gamma_{1}\right), \\
& D_{ \pm}=\frac{1}{\sqrt{2}}\left(D_{0} \pm D_{1}\right),
\end{aligned}
$$

and the $\gamma_{ \pm}$matrices satisfy

$$
\gamma_{ \pm}^{2}=0, \quad\left\{\gamma_{+}, \gamma_{-}\right\}=2
$$


In order to carry out the hamiltonian formulation, we are forced to choose a time coordinate, which is usually chosen as $x^{+}$. Thus the canonical momenta are

$$
\begin{aligned}
\pi_{+}^{a} & =\frac{\delta L}{\delta \partial_{+} A_{+}^{a}}=0 \\
\pi_{-}^{a} & =\frac{\delta L}{\delta \partial_{+} A_{-}^{a}}=F_{+-}^{a}, \\
P^{r} & =\frac{\delta L}{\delta \partial_{+} \psi^{r}}=i \bar{\psi}^{r} \gamma_{-}, \\
\bar{P}^{r} & =\frac{\delta L}{\delta \partial_{+} \bar{\psi}^{r}}=0
\end{aligned}
$$

where $L=\int d x_{-} \mathcal{L}$.

Observing (2.8) one can see that there are three primary constraints, namely

$$
\begin{aligned}
& \pi_{+}^{a}=0, \\
& \chi^{r}=P^{r}-i \bar{\psi}^{r} \gamma_{-}=0, \\
& \bar{\chi}^{r}=\bar{P}^{r}=0,
\end{aligned}
$$

and which must be preserved in time $x^{+}$.

The total hamiltonian can then be computed, with the result

$$
\begin{aligned}
H_{T}= & \int d x_{-}\left[\frac{1}{2}\left(\pi_{-}^{a}\right)^{2}+\pi_{-}^{a} \partial_{-} A_{+}^{a}+g \pi_{-}^{a} f^{a b c} A_{-}^{b} A_{+}^{c}+g \bar{\psi}^{r} \gamma_{-} \psi^{r} A_{+}^{a} T^{a}\right. \\
& \left.-i \bar{\psi}^{r} \gamma_{+} D_{-} \psi^{r}-m \bar{\psi}^{r} \psi^{r}+u_{0}^{a} \pi_{+}^{a}+u_{1}^{r} \chi^{r}+u_{2}^{r} \bar{\chi}^{r}\right]
\end{aligned}
$$

where $u_{0}^{a}, u_{1}^{r}$ and $u_{2}^{r}$ are Lagrange multipliers.

One can see that the preservation in time of $\pi_{+}^{a}$ implies the secondary constraint

$$
G^{a}=\partial_{-} \pi_{-}^{a}+g \pi_{-}^{c} f^{a b c} A_{-}^{b}+g \bar{\psi}^{r} \gamma_{-} \psi^{r} T^{a},
$$

and that the other constraints $\left(\chi^{r}, \bar{\chi}^{r}\right)$ do not generate new constraints.

A straightforward analysis shows that the constraints $G^{a}, \chi^{r}$ and $\bar{\chi}^{r}$ are second class while $\pi_{+}^{a}$ is first class. On the other hand, a simple inspection shows also that $\left(G^{a}, \chi^{r}, \bar{\chi}^{r}\right)$ 
are not a minimal number of second class constraints. The minimal set is found by combining appropiately $G^{a}, \chi^{r}$ and $\bar{\chi}^{r}$, and it is straightforward to verify that this set is

$$
\begin{aligned}
& \Omega_{0}^{a}=\pi_{+}^{a}, \\
& \Omega_{1}^{a}=G^{a}+i\left(\bar{\chi}^{r} T^{a} \psi^{r}+\bar{\psi}^{r} T^{a} \chi^{r}\right), \\
& \chi^{r}=P^{r}-i \bar{\psi}^{r} \gamma_{-}, \\
& \bar{\chi}^{r}=\bar{P}^{r},
\end{aligned}
$$

where $\left(\Omega_{0}^{a}, \Omega_{1}^{a}\right)$ and $\left(\chi^{r}, \bar{\chi}^{r}\right)$ are first and second class constraints respectively.

The first class constraints satisfy the algebra

$$
\left\{\Omega_{\mu}^{a}\left(x_{-}\right), \Omega_{\nu}^{b}\left(x_{-}^{\prime}\right)\right\}=0
$$

while the non-vanishing Poisson bracket between $\chi^{r}$ and $\bar{\chi}^{r}$ are

$$
\left\{\chi_{\alpha}^{r}\left(x_{-}\right), \bar{\chi}_{\beta}^{r^{\prime}}\left(x_{-}^{\prime}\right)\right\}=-i\left(\gamma_{-}\right)_{\beta \alpha} \delta^{r r^{\prime}} \delta\left(x_{-}-x_{-}^{\prime}\right),
$$

where $\alpha, \beta$ are spinorial indices.

In order to eliminate the second class constraints we define the usual Dirac bracket. In this case the non-vanishing Dirac brackets between the canonical variables are

$$
\begin{aligned}
& \left\{A_{+}^{a}\left(x_{-}\right), \pi_{+}^{b}\left(x_{-}^{\prime}\right)\right\}_{D B}=\delta\left(x_{-}-x_{-}^{\prime}\right) \delta^{a b}=\left\{A_{-}^{a}\left(x_{-}\right), \pi_{-}^{b}\left(x_{-}^{\prime}\right)\right\}_{D B}, \\
& \left\{\psi_{\alpha}^{r}\left(x_{-}\right), \bar{\psi}_{\beta}^{r^{\prime}}\left(x_{-}^{\prime}\right)\right\}_{D B}=\left(\gamma_{-}\right)_{\beta \alpha}^{-1} \delta^{r r^{\prime}} \delta\left(x_{-}-x_{-}^{\prime}\right), \\
& \left\{\psi_{\alpha}^{r}\left(x_{-}\right), P_{\beta}^{r^{\prime}}\left(x_{-}^{\prime}\right)\right\}_{D B}=\delta^{r r^{\prime}} \delta^{a b} \delta\left(x_{-}-x_{-}^{\prime}\right), \\
& \left\{\bar{\psi}_{\alpha}^{r}\left(x_{-}\right), \bar{P}_{\beta}^{r^{\prime}}\left(x_{-}^{\prime}\right)\right\}_{D B}=\delta^{r r^{\prime}} \delta^{a b} \delta\left(x_{-} x_{-}^{\prime}\right) .
\end{aligned}
$$

The set of equations (2.10), (2.12-2.15) defines completely the canonical structure of the theory. The next step is to fix the gauge in order to quantize the theory. 


\section{Gauge Fixing and the Path Integral Quantization}

In this section we discuss the quantization of the previous model following the path integral approach. There are several reasons that justify this study: (i) to our knowledge the quantization of $Q C D_{2}$ in the light-front frame following the path integral approach has never been discussed before; (ii) this study could throw some light into the derivation of the Feynman rules in the light-front quantization method and the influence of the zero modes; (iii) the path integral approach could allow for the inclusion of new fields that could simplify the perturbative structure of the theory.

With these facts in mind, in this section we try to clarify the problem of gauge fixing and path integral quantization of $Q C D_{2}$ in the light-front frame.

\section{Gauge Fixing}

The gauge freedom is reflected from the hamiltonian point of view in the presence of first class constraints. In the problem at hand, we have two first class constraints (eqs. $(2.12 \mathrm{a}-\mathrm{b}))$ and, as a consequence, two conditions are necessary in order to fix completely the gauge freedom. Thus, we can start by imposing the following condition as gauge fixing

$$
\Omega_{2}^{a}=A_{-}^{a}=0,
$$

which is known as light-cone gauge and which must be imposed as a new constraint of the theory. Following Dirac's method [8], (3.1) must be preserved in time, i.e.

$$
\partial_{+} \Omega_{2}^{a}=\left\{\Omega_{2}^{a}, H_{T}\right\}=0 .
$$

Computing (3.2), we find that this consistency condition implies the new constraint

$$
\Omega_{3}^{a}=\pi_{-}^{a}+\partial_{-} A_{+}^{a}+g f^{a b c} A_{-}^{b} A_{+}^{c}=0 .
$$

The conditions (3.1) and (3.3) fix completely the gauge freedom. In fact, computing the Poisson algebra we find that the non-vanishing brackets between the first class constraints and the gauge conditions are

$$
\left\{\Omega_{0}^{a}\left(x_{-}\right), \Omega_{3}^{b}\left(x_{-}^{\prime}\right)\right\}=\left(\delta^{a b} \partial_{-}-g f^{a b c} A_{-}^{c}\right) \delta\left(x_{-}-x_{-}^{\prime}\right),
$$




$$
\begin{gathered}
\left\{\Omega_{1}^{a}\left(x_{-}\right), \Omega_{3}^{b}\left(x_{-}^{\prime}\right)\right\}=g f^{a b c}\left(A_{+}^{c} \partial_{-} \delta\left(x_{-}-x_{-}^{\prime}\right)+\partial_{-} A_{+}^{c} \delta\left(x_{-}-x_{-}^{\prime}\right)\right) \\
+g f^{a b c} \pi_{-}^{c} \delta\left(x_{-}-x_{-}^{\prime}\right)+g^{2} f^{a f c} f^{b g c} A_{-}^{f} A_{+}^{g} \delta\left(x_{-}-x_{-}^{\prime}\right)
\end{gathered}
$$

which is a second class constraint algebra.

The question now is: are there other alternative gauge fixing conditions besides $A_{-}^{a}=$ 0 ? . The answer to this question is, of course, yes, although the correct way to implement other possible gauge fixing conditions in the light front-frame is not trivial.

One could try to find, for instance, the analogous of the gauge fixing in a covariant gauge theory, but this procedure does not work here. Indeed, this can be verified by constructing the analogous of the Lorentz gauge $\partial_{\mu} A^{a \mu}=0$ in the light-front

$$
\partial_{-} A_{+}^{a}+\partial_{+} A_{-}^{a}=0,
$$

but a simple analysis shows that this condition does not fix the gauge freedom. In fact, it can be shown that (3.5) is not a true gauge condition because when preservation in time is imposed, we cannot generate a new constraint fixing the remaining gauge symmetry.

The same occurs when we consider the analogous of the axial gauge $n^{\mu} A_{\mu}^{a}=0$ in the light-front

$$
n_{-} A_{+}^{a}+n_{+} A_{-}^{a}=0 .
$$

A possible solution to this problem consists in modifying slightly the previous gauge conditions. Using (3.5) and (3.6) one can see that the unique possible choices for the above gauge conditions are ${ }^{2}$

$$
\begin{gathered}
\left.\partial_{+} A_{-}^{a}=0 \quad \text { (Lorentz gauge }\right) \\
\left.n_{+} A_{-}^{a}=0 \quad \text { (Axial gauge }\right) .
\end{gathered}
$$

The hamiltonian analysis shows, however, that equations (3.7) and (3.8), although they are two independent gauge conditions that fix completely the gauge freedom, are contained in (3.1). This last point can be shown by computing the analogous of (3.2) using (3.7) and (3.8). This calculation gives

$$
\partial_{+} \Omega_{3}^{a}=0=n_{+} \Omega_{3}^{a},
$$

${ }^{2}$ The other possible choices $\partial_{-} A_{+}^{a}=0$ for the Lorentz gauge and $n_{-} A_{+}^{a}=0$ for the axial gauge do not fix the gauge freedom. 
and, as a consequence, to impose (3.7) or (3.8) is formally the same condition (3.1).

This last result means that in two dimensions in the light-front frame, the light-cone gauge (3.1) contains a complete family of gauge conditions that simplify the canonical analysis. $^{3}$ This last point is another advantage of the light-front approach.

\section{$\underline{\text { Path Integral Quantization }}$}

The quantization in this case must be performed using the modication introduced by Senjanovic [10] because there are second class constraints.

The generating functional is

$$
\begin{aligned}
Z= & \int \mathcal{D} \pi_{+}^{a} \mathcal{D} A_{+}^{a} \mathcal{D} \pi_{-}^{a} \mathcal{D} A_{-}^{a} \mathcal{D} \bar{\psi}^{r} \mathcal{D} \psi^{r} \mathcal{D} P^{r} \mathcal{D} \bar{P}^{r} \times \\
& \operatorname{det}\left\|M^{a b}\right\| \operatorname{det}\left\|\left\{\chi_{\alpha}, \chi_{\beta}\right\}\right\|^{\frac{1}{2}} \delta\left(\Omega_{0}^{a}\right) \delta\left(\Omega_{1}^{a}\right) \delta\left(\Omega_{2}^{a}\right) \delta\left(\Omega_{3}^{a}\right) \delta\left(\chi^{r}\right) \delta\left(\bar{\chi}^{r}\right) \\
& \exp \left[i \int d x _ { + } d x _ { - } \left(\pi_{+}^{a} \partial_{+} A_{+}^{a}+\pi_{-}^{a} \partial_{+} A_{-}^{a}+P^{r} \partial_{+} \psi^{r}+\bar{P}^{r} \partial_{+} \bar{\psi}^{r}\right.\right. \\
& -\frac{1}{2}\left(\pi_{-}^{a}\right)^{2}-\pi_{-}^{a} \partial_{-} A_{+}^{a}-g \pi_{-}^{a} f^{a b c} A_{-}^{b} A_{+}^{c}-g \bar{\psi}^{r} \gamma_{-} T^{a} \psi^{r} A_{+}^{a} \\
& \left.\left.+i \bar{\psi}^{r} \gamma_{+} D_{-} \psi^{r}-m \bar{\psi}^{r} \psi^{r}\right)\right] .
\end{aligned}
$$

Using (3.4), the first determinant can be explicitly computed

$$
\operatorname{det}\left\|M^{a b}\right\|=\operatorname{det}\left(\begin{array}{cc}
0 & \kappa^{a b} \\
-\kappa^{a b} & \rho^{a b}
\end{array}\right) \delta\left(x_{-}-x_{-}^{\prime}\right)
$$

where its elements are

$$
\begin{aligned}
& \kappa^{a b}=\delta^{a b} \partial_{-}-g f^{a b c} A_{-}^{c}, \\
& \rho^{a b}=g f^{a b c} \partial_{-} A_{+}^{c}+g f^{a b c} A_{+}^{c} \partial_{-}+g f^{a b c} \pi_{-}^{c}+g^{2} f^{a f c} f^{b g c} A_{+}^{f} A_{-}^{g},
\end{aligned}
$$

while that the second determinant

$$
\operatorname{det}\left\{\chi_{\alpha}^{r}, \bar{\chi}_{\beta}^{r^{\prime}}\right\}=\operatorname{det}\left(\begin{array}{cc}
0 & -i\left(\gamma_{-}\right)_{\beta \alpha}^{-1} \\
-i\left(\gamma_{-}\right)_{\alpha \beta} & 0
\end{array}\right) \delta\left(x_{-}-x_{-}^{\prime}\right) \delta^{r r^{\prime}}
$$

3 Recently there has been some discussion about the problem of fixing the residual gauge in light-front $Q C D_{4}[\mathbf{9}]$. In this paper we assume periodic boundary conditions, in which case the zero modes do not have to be considered. 
is a c-number and can be dropped off the path integral as a normalization factor.

Integrating in $\pi_{-}^{a}, \pi_{+}^{a}, A_{-}^{a}, \bar{P}^{r}, P^{r}$ and exponentiating (3.10) in terms of anticommutative ghosts, we find

$$
\begin{aligned}
Z= & \mathcal{N} \int \mathcal{D} A_{+}^{a} \mathcal{D} \bar{\psi}^{r} \mathcal{D} \psi^{r} \mathcal{D} \text { (ghosts) } \delta\left[-\partial_{-}^{2} A_{+}^{a}+g \bar{\psi}^{r} \gamma_{-} T^{a} \psi^{r}\right] \times \\
& \exp \left[i \int d x _ { + } d x _ { - } \left(\frac{1}{2}\left(\partial_{-} A_{+}^{a}\right)^{2}+i \bar{\psi}^{r} \gamma_{+} \partial_{-} \psi^{r}+i \bar{\psi}^{r} \gamma_{-} \partial_{+} \psi^{r}\right.\right. \\
& \left.\left.-g \bar{\psi}^{r} \gamma_{-} T^{a} \psi^{r} A_{+}^{a}-m \bar{\psi}^{r} \psi^{r}+(\text { ghosts })\right)\right],
\end{aligned}
$$

where $\mathcal{N}$ is a normalization factor.

Using the antisymmetry of the structure constants and the anticommutative character of the ghosts, it is easy to see that there is no coupling between the ghosts and the gauge fields and that the integration in the ghosts fields is trivial. This result is a consequence of a general statement that establishes that all axial like gauges are free of interactions with the ghosts [11]. Having this fact in mind, (3.12) becomes

$$
\begin{aligned}
Z= & \mathcal{N}^{\prime} \int \mathcal{D} A_{+}^{a} \mathcal{D} \bar{\psi}^{r} \mathcal{D} \psi^{r} \delta\left[-\partial_{-}^{2} A_{+}^{a}+g \bar{\psi}^{r} \gamma_{-} T^{a} \psi^{r}\right] \times \\
& \exp \left[i \int d x _ { + } d x _ { - } \left(\frac{1}{2}\left(\partial_{-} A_{+}^{a}\right)^{2}+i \bar{\psi}^{r} \gamma_{+} \partial_{-} \psi^{r}+i \bar{\psi}^{r} \gamma_{-} \partial_{+} \psi^{r}\right.\right. \\
& \left.\left.-g \bar{\psi}^{r} \gamma_{-} T^{a} \psi^{r} A_{+}^{a}-m \bar{\psi}^{r} \psi^{r}\right)\right] .
\end{aligned}
$$

This formula gives the path integral version of $Q C D_{2}$.

The constraint (Gauss's law):

$$
\partial_{-}^{2} A_{+}^{a}-J_{-}^{a}=0
$$

with $J_{-}^{a}=g \bar{\psi}^{r} \gamma_{-} T^{a} \psi^{r}$, physically can be understood as follows. In two dimensions there only exists an electric field, which is given by $E^{a}=\partial_{-} A_{+}^{a}$. Using this fact and equation (3.14), $E^{a}$ is given by

$$
E^{a}\left(x_{-}\right)=\int d x_{-}^{\prime} \epsilon\left(x_{-}-x_{-}^{\prime}\right) J_{-}^{a}\left(x_{-}^{\prime}\right),
$$

where $\epsilon\left(x_{-}-x_{-}^{\prime}\right)$ is the sign function. In order to see what this result means, let us assume for the moment that the quarks are point-like. Thus $J_{-}^{a}\left(x_{-}\right)$can be written as

$$
J_{-}^{a}\left(x_{-}\right)=\sum_{\alpha=1}^{N} q_{\alpha} \delta\left(x_{-}-x_{-}^{\alpha}\right) T^{a}
$$


where $q_{\alpha}$ is the quark charge and $x_{-}^{\alpha}$ is the place where the $\alpha$-th quark is localized. Using (3.16), the electric field becomes

$$
E^{a}\left(x_{-}\right)=\sum_{\alpha=1}^{N} q_{\alpha} \epsilon\left(x_{-}-x_{-}^{\alpha}\right) T^{a}
$$

Therefore the electric field between the particles is unable to spread out and the quarks are confined. The reader should notice that confinement is present irrespective of the (non)abelian character of the gauge field [12].

The next step is to integrate the $A_{+}^{a}$ field. Using the identity

$$
\int \mathcal{D} \phi \delta(A \phi+B) e^{i \int d x(\phi C \phi+D \phi)}=e^{i \int d x\left(\frac{B}{A} C \frac{B}{A}-\frac{D}{A} B\right)},
$$

where $A, B, C, D$ are operators (with inverse), equation (3.13) becomes

$$
\begin{aligned}
Z= & \mathcal{N} \int \mathcal{D} \bar{\psi}^{r} \mathcal{D} \psi^{r} \exp \left[i \int d x _ { + } d x _ { - } \left(\bar{\psi}^{r}\left(i \gamma_{+} \partial_{-}+i \gamma_{-} \partial_{+}-m\right) \psi^{r}\right.\right. \\
& \left.\left.-\frac{1}{4} \int d x_{-}^{\prime} J_{-}^{a}\left(x_{-}\right)\left|x_{-}-x_{-}^{\prime}\right| J_{-}^{a}\left(x_{-}^{\prime}\right)\right)\right]
\end{aligned}
$$

where $J_{-}^{a}\left(x_{-}\right)=g \bar{\psi}^{r}\left(x_{-}\right) \gamma_{-} T^{a} \psi^{r}\left(x_{-}\right)$and $\left|x_{-}-x_{-}^{\prime}\right|$ is the propagator of the gluon field obtained by inverting the operator $\partial_{-}^{2}$.

We would like to add a few sentences about the procedure used here for inverting the operator $\partial_{-}^{2}$. The central observation consists in noticing that the invertion of $\partial_{-}^{2}$ is a similar mathematical problem to the calculation of the propagator of a massless relativistic particle [13] moving in a one-dimensional space. Following this analogy, $\frac{1}{\partial_{-}^{2}}$ can be represented by the integral

$$
\int_{0}^{\infty} d T T^{-\frac{D}{2}} \exp \left[-\frac{\Delta \vec{x}^{2}}{2 T}\right]
$$

where for generality, we are supposing a $D$-dimensional operator and $\Delta \vec{x}=\vec{x}-\vec{x}^{\prime}$.

The integral (3.20), however, is infrared divergent and it is necessary to perform a regularization for extracting a finite result. In order to regularize, we replace (3.20) by

$$
\int_{0}^{\infty} d T T^{-\frac{D}{2}} \exp \left[-\frac{(\Delta \vec{x})^{2}}{2 T}-\frac{m^{2}}{2} T\right]
$$


where $m^{2}$ is a massive regulator that is put equal to zero at the end of the calculation.

The integral (3.21) can be computed explicitly and gives the following result

$$
\sim\left[\frac{\Delta|\vec{x}|}{m}\right]^{\frac{1}{2}-\frac{D}{4}} K_{\frac{1}{2}-\frac{D}{4}}(m \Delta|\vec{x}|)
$$

where $K_{\frac{1}{2}-\frac{D}{4}}$ is the Bessel function.

The massless limit corresponds to the replacement of the Bessel function by its asym-

totic behaviour when $m \rightarrow 0$, i.e. $\sim[m \Delta|\vec{x}|]^{\frac{1}{2}-\frac{D}{4}}$. Then the limit becomes regular and we find for $D=1$ the result that appears in (3.19).

Equation (3.19) is the starting point for the perturbative and non-perturbative evaluation of quantum corrections of $Q C D_{2}$. Non-perturbati-vely this formula was used in reference [14] in order to derive the t'Hooft equation for the bound states in the large- $\mathrm{N}$ limit.

From the canonical point of view, the effective action that appears in equation (3.19) was used by the authors of reference [15] for the numerical study of light-front quantized $Q C D_{2}$.

Finally, we should mention that the Higgs mechanism in light-front quantized field theory was also considered in reference [16].

\section{Conclusions}

We summarize our results. In this paper we have studied the canonical structure of $Q C D_{2}$ in the light-front frame and have also quantized the theory using the path integral formalism.

We have discussed some problems with the gauge fixing procedure and we have shown how the Lorentz or axial like gauges in the light-front are contained in the light-cone gauge.

The path integral quantization was performed using the Senjanovic method due to the existence of second class constraints and an effective field theory for the fermionic fields that corresponds to a Nambu-Jona Lasinio model was obtained. This effective field theory is the starting point for some approachs to $Q C D_{2}$. 


\section{REFERENCES}

[1] For a review and references, see S. J. Brodsky, G. McCastor, H.-C. Pauli and S. S. Pinsky, Particle World 3 (1993) 109

[2] P. A. M. Dirac, Rev. of Mod. Phys. 21 (1949) 392; S. Weinberg, Phys. Rev. 50 (1966) 1313.

[3] H. C. Pauli and S. J. Brodsky, Phys. Rev. D32 (1985) 1993; ibid. D32 (1985) 2001.

[4] R. J. Perry, A. Harindranath and K. G. Wilson, Phys. Rev. Lett. 65 (1990) 2959; D. Moustaki, S. Pinsky, J. Shigenitsu and K. G. Wilson, Phys. Rev. D43 (1991) 3411.

[5] G. t'Hooft, Nucl. Phys. B75 (1974) 461.

[6] D. Moustaki, Phys. Rev. D42 (1990) 1184.

[7] F. Lentz, M. Thies, S. Levit and K. Yazaki, Ann. of Phys. 208 (1991) 1; T. Heinzl, S. Krusche and E. Werner, Phys. Lett. B275 (1992) 410; K. Hornbostel, Phys. Rev. D45 (1992) 3781.

[8] See for example: K. Sundermeyer, Constrained Hamiltonian Systems, Springer Verlag, 1984.

[9] W.-M. Zhang and A. Harindranath, Phys. Lett. B314 (1993) 223.

[10] P. Senjanovic, Ann. of Phys. 100 (1976) 227.

[11] See for example: G. Leibbrandt, Rev. of Mod. Phys. 59 (1987) 1067; W. Konetschny, Phys. Lett. B90 (1980)263.

[12] S. Coleman, R. Jackiw and L. Susskind, Ann. of Phys. 93 (1975) 267; S. Coleman, ibid. 101 (1976) 239.

[13] J. Schwinger, Phys. Rev. 209(1951) 749.

[14] W. R. Gutiérrez, Nucl. Phys. B176 (1980) 185.

[15] K. Hornbostel, S. J. Brodsky and H. C. Pauli, Phys. Rev. D41 (1990) 3814.

[16] P. P. Srivastava, preprints CBPF-010/92 and CBPF-004/92. 